\title{
Electrospun quercetin-loaded zein nanoribbons
}

\author{
Xiao-Yan Li ${ }^{\mathrm{a}}$, Chen-Jie Shi ${ }^{\mathrm{b}}$, Deng-Guang $\mathrm{Yu}^{\mathrm{a},{ }^{*}}$, Yao-Zu Liao ${ }^{\mathrm{c}}$ and Xia Wang ${ }^{\mathrm{a},{ }^{*}}$ \\ ${ }^{a}$ School of Materials Science \& Engineering, University of Shanghai for Science and Technology, \\ Shanghai 200093, China \\ ${ }^{b}$ School of Environment and Architecture, University of Shanghai for Science and Technology, \\ Shanghai 200093, China \\ ${ }^{c}$ School of Chemistry, University of Bristol, Bristol, England BS8 1TS, UK
}

\begin{abstract}
This study investigates quercetin-loaded zein nanoribbons, which were fabricated using different types of electrospinning processes. Using ethanol aqueous solutions as sheath fluids, the widths of quercetin-loaded zein nanoribbons $(D, \mathrm{~nm})$ could be manipulated simply through the adjustment of water contents $(C)$ in the sheath fluids according to an equation of $D=958-8.01 C(r=0.9977)$, as indicated by the field emission scanning electron microscopic observations. X-ray diffraction and attenuated total reflectance Fourier transform infrared analysis suggested that the quercetin presented in the zein nanoribbons in an amorphous state due to their high compability resulted from hydrogen bonds. In vitro dissolution tests verified that nanoribbons from the coaxial process and single fluid process could provide drug sustained release profiles via a typical Fickian diffusion mechanism, and the former exhibited better performance than the later in terms of small initial burst effect and leveling-off release. Coaxial electrospinning with solvents can expand the capability of electrospinning in generating nanoproducts and provide a way for improving the nanoproducts' quality and functional performance.
\end{abstract}

Keywords: Zein, quercetin, nanoribbons, coaxial electrospinning, drug delivery

\section{Introduction}

Coaxial electrospinning can apparently not only be used to produce core-shell and hollow nanofibers but rather also to improve the quality of monolithic nanofibers [1]. To demonstrate this, a polymer solution was fed to the core die whereas salt solutions or appropriately selected solvent mixtures were used as shell fluids. Exploiting these approach fibers with smaller diameters, smoother surfaces and with more uniform structures are reported to become accessible [1-4].

Zein is a mixture of proteins with different molecular weights in corn gluten meal. Apart from its favorable biodegradability and biocompatibility, it has low hydrophilicity, good elasticity, and film-forming capabilities [5,6]. Zein is used in the manufacture of a wide variety of commercial products, including coatings for food, excipients for pharmaceutics, and clothing. Most recently, its

\footnotetext{
* Corresponding authors: Deng-Guang Yu, School of Materials Science \& Engineering, University of Shanghai for Science and Technology, Shanghai 200093, China. Tel.: +86-021-55274069; Fax:+86-021-55270632; E-mail: ydg017@gmail.com.

Xia Wang, School of Materials Science \& Engineering, University of Shanghai for Science and Technology, Shanghai 200093, China. Tel.:+86-021-55271686; Fax:+86-021-55270632; E-mail: wangxia@usst.edu.cn.
} 
electrospinnability into nanofiber mats for biomedical applications has been investigated [7-9]. Today in pharmaceutical field, nano drug delivery systems have drawn increasing attention [10-13]. Based on the unique property of zein, its electrospun nanofibers may be potential novel drug delivery systems for providing sustained drug release profile [14,15].

Quercetin is a plant pigment (flavonoid). It is found in many foods and plants, such as red wine, onions, apples, green tea, berries, and Ginkgo biloba. Quercetin is used for treating conditions of the heart and blood vessels including "hardening of the arteries" (atherosclerosis), heart disease, high cholesterol, and circulation problems. It is also used for cataracts, diabetes, peptic ulcer, hay fever, schizophrenia, asthma, inflammation, gout, viral infections, chronic fatigue syndrome, preventing cancer, for treating chronic infections of the prostate, and for increasing endurance and improving athletic performance. Sustained release of quercetin after oral administration is highly desired for the convenience of patients and therapeutic effects $[16,17]$.

In this study, with quercetin as the model pharmaceutical active ingredient, we evaluated the preparation and characterization of medicated zein composite ribbons using a coaxial electrospinning. The coaxial process involved using only unspinnable ethanol aqueous solutions as the sheath fluid to facilitate a smooth and continuous electrospinning process and to produce nanofibers with higher quality and improved functional performance.

\section{Experimental}

\subsection{Materials}

Quercetin (purity $>98 \%$, No. MUST-12072505) was purchased from the Beijing Aoke Biological Technology Co., Ltd. (Beijing, China). Zein (purity, 98\%) was obtained from Aldrich (Milwaukee, WI, USA). Acetic acid (AC) and anhydrous ethanol were provided by Sinopharm Chemical Reagent Co., Ltd. (Shanghai, China). All other chemicals used were of analytical grade, and water was doubly distilled immediately before use.

\subsection{Modified coaxial electrospinning}

The core electrospinnable mixed solutions of zein and quercetin were prepared by dissolving $28 \mathrm{~g}$ of zein and $3 \mathrm{~g}$ of quercetin in a mixture of $\mathrm{AC}$ and ethanol with a volume ratio of 25:75. Anhydrous ethanol and ethanol aqueous solutions with different content of water were explored as sheath fluids. Two syringe pumps (KDS100 and KDS200, Cole-Parmer, IL, USA) and a high-voltage $(60 \mathrm{kV} / 2 \mathrm{~mA})$ power supply (ZGF2000, Shanghai Sute Corp., Shanghai, China) were used for the coaxial electrospinning. All electrospinning processes were carried out under ambient conditions $\left(24 \pm 4^{\circ} \mathrm{C}\right.$ with relative humidity of $61 \pm 5 \%$ ). A homemade concentric spinneret was used to conduct both single-fluid (with the sheath fluid adjusted to $0 \mathrm{~mL} / \mathrm{h}$ ) and coaxial electrospinning. The electrospinning process was recorded using a digital video recorder (PowerShot A490; Canon, Tokyo, Japan). Following some optimization, the applied voltage, fibers collected distance on aluminum foil and the sheath-to-core flow rate ratio were fixed at $15 \mathrm{kV}$, a distance of $20 \mathrm{~cm}$ and 0.2 respectively. All other parameters are listed in Table 1. 
Table 1

Parameters of the electrospinning processes and their products

\begin{tabular}{|c|c|c|c|c|}
\hline No. & Process & $\begin{array}{l}\text { Water content in } \\
\text { sheath fluid }{ }^{b}\end{array}$ & Fiber Morphology & $\begin{array}{l}\text { Width }^{\mathrm{c}} \\
(\mathrm{nm})\end{array}$ \\
\hline $\mathrm{R} 1$ & Single & -- & Linear ribbon & $1140 \pm 230$ \\
\hline $\mathrm{R} 2$ & \multirow{4}{*}{ Coaxial $^{\mathrm{a}}$} & $0 \%$ & Linear ribbon & $970 \pm 180$ \\
\hline $\mathrm{R} 3$ & & $15 \%$ & Linear ribbon & $830 \pm 120$ \\
\hline $\mathrm{R} 4$ & & $30 \%$ & Linear ribbon & $720 \pm 110$ \\
\hline R5 & & $50 \%$ & Spindles-on-a-line & $560 \pm 130$ \\
\hline
\end{tabular}

\subsection{Characterization}

\subsubsection{Morphology}

The morphology of the fiber mats was assessed using field emission scanning electron microscopy (FESEM) with S-4800 (Hitachi, Tokyo, Japan). Prior to the examination, the samples were platinum sputter-coated under a nitrogen atmosphere to render them electrically conductive. Images were then recorded at an excitation voltage of $10 \mathrm{kV}$. The sizes of the fibers were determined by measuring their widths in FESEM images in more than 100 different places using Image $\mathbf{J}$ software (National Institutes of Health, MD, USA) and were expressed in $\mu \mathrm{m}$ as mean \pm SD. Because the zein nanofibers were in the form of ribbons, thus hereinafter nanofibers and nanoribbons are equally used. The topography of the quercetin particles was observed under cross-polarized light using an XP-700 polarized optical microscope (Shanghai Changfang Optical Instrument Co., Ltd).

\subsubsection{Physical status and compatibility}

X-Ray Diffraction (XRD) analysis was performed using a D/Max-BR diffractometer (RigaKu, Japan) with $\mathrm{Cu} \mathrm{K} \alpha$ radiation in the $2 \theta$ range of $5-60^{\circ}$ at $40 \mathrm{mV}$ and $300 \mathrm{~mA}$. Attenuated total reflectance Fourier transform infrared (ATR-FTIR) analysis was carried out on a Nicolet-Nexus 670 FTIR spectrometer (Nicolet Instrument Corporation, Madison, USA) over the range of $500-4000 \mathrm{~cm}^{-1}$ and at $2 \mathrm{~cm}^{-1}$ of resolution. The test samples included the individual ingredients and their ribbons.

\subsubsection{In vitro dissolution tests}

In vitro dissolution tests were carried out according to Method II, as described in Chinese Pharmacopoeia (2005 Ed.), a paddle method for which an RCZ-8A dissolution apparatus (Tianjin University Radio Factory, Tianjin, China) was used. Two hundred milligrams of fibers R1 and R2 was put into $900 \mathrm{~mL}$ of physiological saline $(0.9 \mathrm{wt} \%)$ at $37 \pm 1^{\circ} \mathrm{C}$ and the instrument was set to $50 \mathrm{rpm}$, providing sink conditions in which $C<0.2 C_{\mathrm{s}}$. At predetermined time points, 5 -mL samples were withdrawn from the dissolution medium and replaced with fresh medium to maintain a constant volume. After filtration through a $0.22-\mu \mathrm{m}$ membrane (Millipore, MA, USA) and appropriate dilution with physiological saline, samples were analyzed at $\lambda_{\max }=371 \mathrm{~nm}$ using a UV/vis spectrophotometer (UV-2102PC; Unico Instrument Co. Ltd., Shanghai, China). The cumulative amount of quercetin released at each time point was back-calculated from the data obtained against a predetermined 


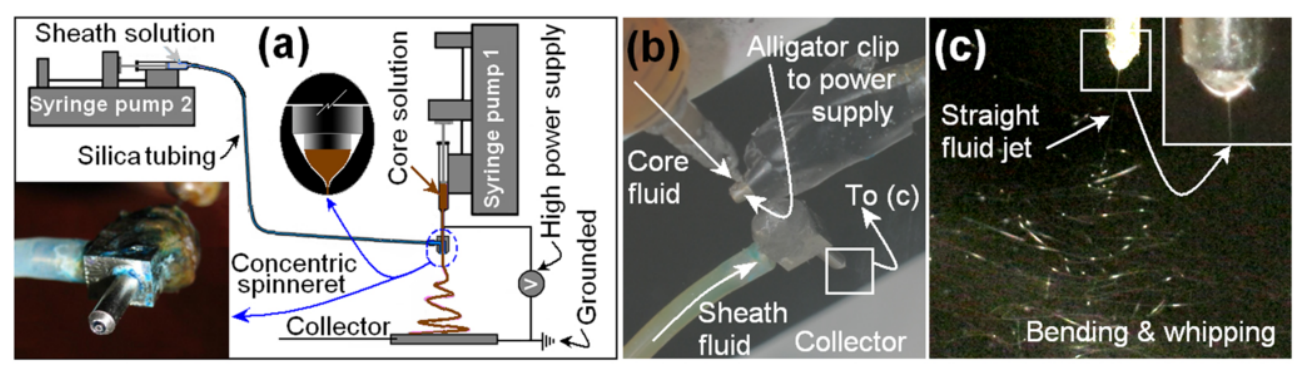

Fig. 1. The modified coaxial electrospinning: (a) a diagram of the coaxial electrospinning process and the inset is a close-up view of the homemade concentric spinneret, (b) the connection of the concentric spinneret with the syringe pumps and the power supply, (c) a typical coaxial electrospinning process under an applied voltage of $15 \mathrm{kV}$ and a sheath fluid composed of $30 \%$ water for nanoribbons R4, the inset is a picture of the compound Taylor cone.

calibration curve. All measurements were carried out six times, and the results were expressed in percentage as mean $\pm \mathrm{SD}$.

\section{Results and discussion}

\subsection{Modified coaxial electrospinning process}

A schematic diagram of the modified coaxial electrospinning process is shown in Figure 1a. A homemade concentric spinneret was developed to carry out the modified process (the inset of Figure 1a). When the modified coaxial electrospinning was carried out to prepare the composite nanofibers, two syringe pumps were exploited to drive the sheath and core fluids independently. An alligator clip was used to connect the inner stainless steel capillary with the high power supply (Figure 1b). With ethanol aqueous solution ( $30 \%$ water) as a sheath fluid and under a sheath-to-core flow rate ratio of 0.2 , the arrangement produced a typical fluid jet trajectory, in which a Taylor cone followed by a straight fluid jet and then a bending and whipping instability region with loops of increasing size (Figure 1c).

\subsection{Morphologies of the prepared quercetin-loaded zein nanofibers}

Nanofibers obtained from the single fluid electrospinning and the modified coaxial electrospinning and their widths distribution are showed in Figure 2. All the quercetin-loaded nanofibers have a ribbon morphology and good structural uniformity except R5. When only ethanol was exploited as a sheath fluid and under a sheath-to-core flow rate ratio of 0.2 , the nanoribbons' width decreased obviously from $1140 \pm 230 \mathrm{~nm}$ ( R1 ribbons, Figure 2a) to $970 \pm 180 \mathrm{~nm}$ (R2 ribbons, Figure 2b). When the ethanol aqueous solutions were used as sheath fluids and as the content of water in the sheath fluids increased to $15 \%, 30 \%$ and $50 \%$, the resultant nanoribbons $\mathrm{R} 3, \mathrm{R} 4$ and $\mathrm{R} 5$ were progressively narrower (Figures 2c to 2e), with a width of $830 \pm 120 \mathrm{~nm}, 720 \pm 110 \mathrm{~nm}$, and $560 \pm 130 \mathrm{~nm}$, respectively. All had widths smaller than R1 ribbons (Figure 2a) prepared from the core solutions alone using a single fluid electrospinning process. However, naoribbons R5 had some spindles in them, as indicated by "A" in Figure 2e. This should a result from the excessive water in the sheath solutions, which has been reported in a similar research [18]. 


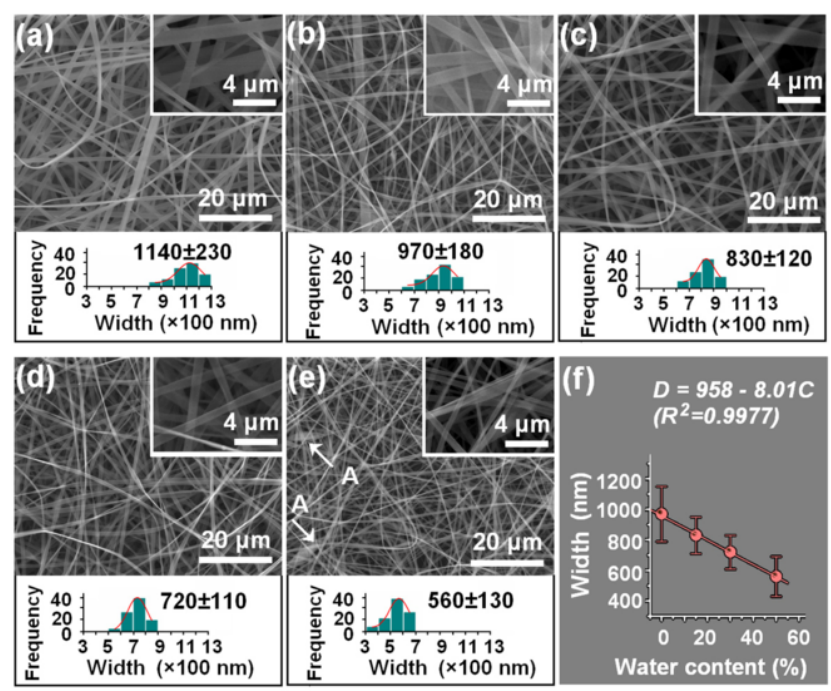

Fig. 2. FESEM images of the ribbons and their width distributions: (a) R1, (b) R2, (c) R3, (d) R4, (e) R5, and (f) the relationship between the widths of ribbon and the water content in the sheath fluid.

Compared to ethanol, water has a higher boiling point and more difficult to evaporate during the electrospinning process. Thus the increase of water in the sheath fluids would mean that the sheath fluids would keep the core jets in a fluid state for a longer time period to be subjected to the electrical drawing, and thus to result in narrower nanoribbons. Linear relationship between the water content in the sheath fluid $(C)$ and the resultant nanoribbons' average widths $(D, \mathrm{~nm})$ was found (Figure $2 \mathrm{f})$. The regressed equation is $D=958-8.01 C$, with a correlation of 0.9977 . The results suggest that the widths of uniform ribbons can be tailored simply through manipulating the water content in the sheath fluid within a suitable range through the modified coaxial electrospinning.

In summary, these results demonstrate that the modified coaxial electrospinning is useful for improving quercetin-loaded zein nanofibers quality in terms of nanofibers' morphology and widths with a reasonable selection of sheath mixed solvent.

\subsection{Physical status of quercetin and its compatibility with matrix zein}

The XRD patterns of quercetin, zein and their nanoribbons R1 and R4 are shown in Figure 3a. Quercetin, a yellowish green powder to the naked eye, comprises polychrome crystals in the form of prisms or needles. The crystals exhibit a rough surface under cross-polarized light (Figure $3 b$ ). The presence of many distinct peaks (Figure 3a) in the XRD pattern of pure quercetin verified the crystalline nature of the material. The pair of diffraction haloes in the diffraction pattern of raw zein powders indicates that the polymer is amorphous. In Figure 3a, the characteristic diffraction peaks of crystalline quercetin are seen to be completely absent in both types of nanoribbons. These results suggest that the quercetin in the nanoribbons R1 and R4 is similarly completely amorphous, regardless of their different electrospinning processes.

The molecular structures of quercetin and zein are shown in Figure 4. Zein molecules possess -NHand $-\mathrm{C}=\mathrm{O}$ groups, suggesting that it could act as both as proton donors and proton acceptor for forming hydrogen bonding. Quercetin molecules possess free $-\mathrm{C}=\mathrm{O}$ and $-\mathrm{OH}$ groups, similarly suggesting that it can also act as proton acceptors and donors in hydrogen bonding. Therefore it can be 


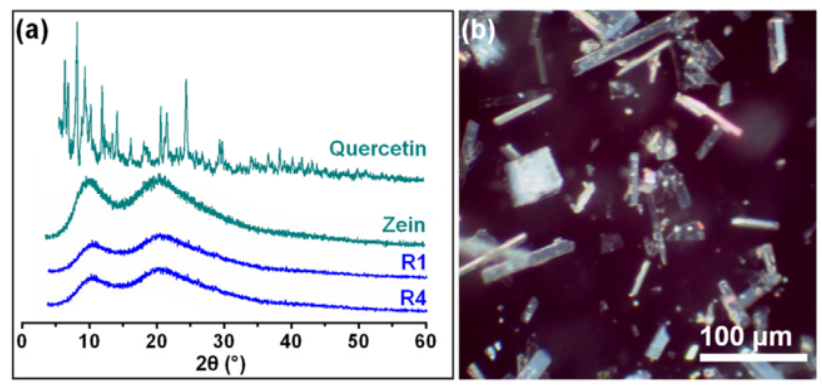

Fig. 3. (a) XRD patterns of the raw materials and their nanoribbons R1 and R4, and (b) crystals of quercetin viewed under cross-polarised light.

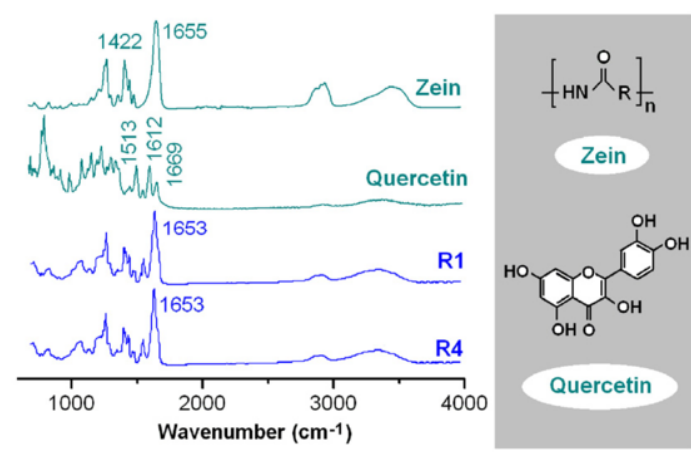

Fig. 4. ATR-FTIR spectra of the raw materials and their nanoribbons R1 and R4, and molecular formula of zein and quercetin.

speculated that hydrogen bonding occurs within the nanoribbons through interactions between zein and quercetin moulecules. Figure 4 shows the ATR-FTIR spectra of zein, quercetin and their nanoribbons R1 and R4. Sharp peaks appear in the spectrum of crystalline quercetin at $1612 \mathrm{~cm}^{-1}$ and $1669 \mathrm{~cm}^{-1}$, representing the stretching vibration of the benzene rings and the $-\mathrm{C}=\mathrm{O}$ groups, respectively. However, none of the quercetin peaks were present in the ATR-FTIR spectra of the nanoribbons R1 and R4. Numerous peaks in the finger region of the quercetin spectrum were also absent in the nanoribbons' spectra. Collectively, these results suggest that the quercetin molecules in the nanoribbons undergo fewer interactions than in pure quercetin, but form extensive hydrogen bonds with the zein molecules. This hydrogen bonding, combined with possible hydrophobic interactions, should provide a stabilizing environment for the structure, yielding a high degree of compatibility between the nanoribbon components, and consequently a homogeneous nano-composite of quercetin and zein.

\subsection{In vitro dissolution tests}

Comparative results for the in vitro dissolution profiles of the nanoribbons R1 from the single fluid electrospinning and R4 from the coaxial electrospinning are shown in Figure 5. Both quercetin-loaded zein ribbons provided similar sustained-release profiles. The quercetin release profiles were analyzed according to the Peppas equation [19]:

$$
Q=k t^{\mathrm{n}}
$$


(a)

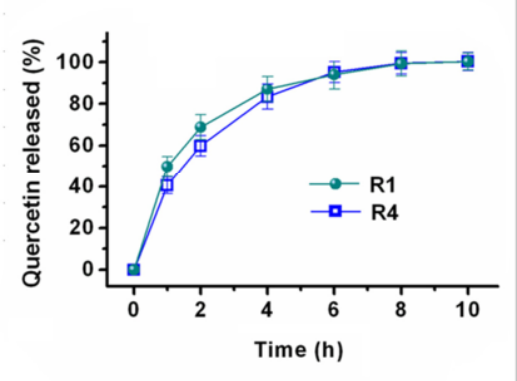

(b) Nanoribbons

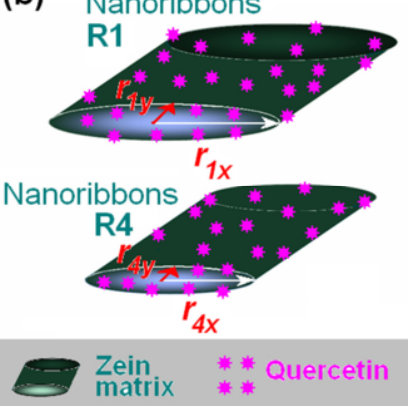

Fig. 5. In vitro drug release profiles of nanoribbons $\mathrm{R} 1$ and $\mathrm{R} 4(\mathrm{n}=6)(\mathrm{a})$ and a sketch about the drug controlled release mechanisms of the nanoribbons (b).

where $Q$ is the drug release percentage, $t$ is the release time, $k$ is a constant reflecting the structural and geometric characteristics of fibers, and $n$ is the release exponent, which is indicative of the drug release mechanism.

The equations for $\mathrm{R} 1$ and $\mathrm{R} 4$ are $\mathrm{Q}=41.8 \mathrm{t}^{0.31}(\mathrm{R}=0.9887)$ and $\mathrm{Q}=50.4 \mathrm{t}^{0.37}(\mathrm{R}=0.9824)$, respectively. The results demonstrated that quercetin was released from the ribbons via a typical Fickian diffusion mechanism, as indicated by a value of the release exponent lower than $0.45(0.31$ for R4 and 0.37 for $\mathrm{R} 1$ ), suggesting that the coaxial process has no influence on the drug release mechanism, which is often determined by the properties of the polymer matrix.

However, a further detailed comparison of the release profiles suggested that the ribbons $\mathrm{R} 4$ from the coaxial process performed better than the ribbons R1 from single fluid electrospinning. First, the former ribbons had a smaller initial burst release effect during the first hour $(40.6 \%)$ than the latter $(49.7 \%)$. The initial burst effect is a common phenomenon for drug-loaded nanofibers resulting from high surface area and porosity, and methods such as post-treatment of cross-linking and coaxial electrospinning to encapsulate drugs in the core part are used to smoothen or eliminate it for improving the sustained-release profile [20]. Second, R4 had a shorter leveling-off period than R1. For the last stage of sustained release, the smaller the amount of unreleased drug and the shorter the leveling-off period were, the better-because drug release in the latter period cannot deliver an effective therapeutic concentration in the blood. Thus, it can be concluded that the ribbons from the modified coaxial electrospinning process (R4) were able to provide a better sustained-release profile than those from the single-fluid process (R1).

As mentioned, quercetin was released from the ribbons through a typical Fickian diffusion mechanism. Typically, the diffusion process consisted of three steps: (1) the water molecules penetrated the inner part of zein nanoribbons gradually, (2) they then dissolved the quercetin molecules hung on the zein molecules, and (3) the free quercetin molecules passively diffused from the ribbons to the dissolution medium. As the drug quercetin was distributed in the zein matrix in an amorphous state, that is, the dissolution of quercetin in water was a fast process, the penetration of water and the diffusion of quercetin determined the apparent dissolution rate of quercetin from the ribbons. Meanwhile, the process would move forward along all directions to the center of the fibers; thus, the quercetin molecules would be freed into the dissolution medium via the shortest way (i.e. the direction of $r_{l y}$ and $r_{4 y}$ in Figure 5b). Although ribbons R1 had a larger average width than R2, they had a similar flat ribbon morphology, and what is important, the R1 fibers had a large size distribution 
of widths, thus they gave a larger initial burst drug release effect and a more severe leveling-off release.

\section{Conclusion}

Quercetin-loaded zein nanoribbons have been successfully prepared using a traditional single fluid electrospinning and also the coaxial electrospinning processes. FESEM results demonstrated that the widths of quercetin-loaded zein nanoribbons $(D, \mathrm{~nm})$ could be manipulated simply through the adjustment of water contents $(C)$ in the sheath fluids according to an equation of $D=958-8.01 C$, with a correlation of 0.9977 . XRD and ATR-FTIR results verified that the quercetin presented in the zein nanoribbons in an amorphous state due to their high compability resulted from hydrogen bonds. In vitro dissolution tests demonstrated that nanoribbons from the coaxial process and single fluid process could provide sustained release profiles of quercetin, and the former exhibited better performance than the later in terms of initial burst effect and leveling-off release. Coaxial electrospinning with solvents can expand the capability of electrospinning in generating nanoproducts and provide a way for improving the nanoproducts' quality and functional performance.

\section{Acknowledgement}

This work was supported by the National Science Foundation of China (Nos. 51373101 and 51373100), the Natural Science Foundation of Shanghai (No. 13ZR1428900) and the Key Project of the Shanghai Municipal Education Commission (Nos. 13ZZ113 and 13YZ074).

\section{References}

[1] S. Agarwal, A. Greiner and J.H. Wendorff, Functional materials by electrospinning of polymers, Prog. Polym. Sci. 38 (2013), 963-991.

[2] D.G. Yu, J.H. Yu, L. Chen, G.R. Williams and X. Wang, Modified coaxial electrospinning for the preparation of high-quality ketoprofen-loaded cellulose acetate nanofibers, Carbohydr. Polym. 90 (2012), 1016-1023.

[3] D.G. Yu, G.R. Williams, X. Wang, X.K. Liu, H.L. Li and S.W.A. Bligh, Dual drug release nanocomposites prepared using a combination of electrospraying and electrospinning, RSC Adv. 3 (2013), 4652-4658.

[4] W. Huang, T. Zou, S. Li, J. Jing, X. Xia and X. Liu, Drug-loaded zein nanofibers prepared using a modified coaxial electrospinning process, AAPS PharmSci.Tech. 14 (2013), 675-681.

[5] L.F. Lai and H.X. Guo, Preparation of new 5-fluorouracilloaded zein nanoparticles for liver targeting, Int. J. Pharm. 404 (2011), 317-323.

[6] K. Kanjanapongkul, S. Wongsasulak and T. Yoovidhya, Prediction of clogging time during electrospinning of zein solution: Scaling analysis and experimental verification, Chem. Eng. Sci. 65 (2010), 5217-5225.

[7] W. Nie, D.G. Yu, C. Branford-White, X.X. Shen and L.M. Zhu, Electrospun Zein/PVP fiber composite and its application in drug delivery, Mater. Res. Innov. 16 (2012), 14-18.

[8] S.K. Tiwari, R. Tzezana, E. Zussman and S.S. Venkatraman, Optimizing partition-controlled drug release from electrospun core-shell fibers, Int. J. Pharm. 392 (2010), 209-217.

[9] H.L. Jiang, P.C. Zhao and K.J. Zhu, Fabrication and characterization of zein-based nanofibrous scaffolds by an electrospinning method, Macromol. Biosci. 7 (2007), 517-525.

[10] R. Fang, C.M.J. Hu, B.T. Luk, W. Gao, J. Copp, Y. Tai, D. O'Connor and L. Zhang, Caner cell membrane-coated nanoparticles for anticancer vaccination and drug delivery, Nano. Lett.14 (2014), 2181-2188.

[11] D.S. Katti, K.W. Robinson, F.K. Ko and C.T. Laurencin, Bioresorbable nanofiber-based systems for wound healing and drug delivery: Optimization of fabrication parameters, J. Biomed. Mater. Res. 70B (2004), 286-296. 
[12] M. Ghosh, A.T.K. Singh, W. Xu, T. Sulchek, L.I. Gordon and R.O. Ryan, Curcumin nanodisks: formulation and characterization, Nanomed. Nanotech. Biol. Med. 7 (2011), 162-167.

[13] A. Sharma, A. Gupta, G. Rath, A. Goyal, R.B. Mathur and S.R. Dhakate, Electrospun composite nanofiber-based transmucosal patch for anti-diabetic drug delivery, J. Mater. Chem. 1B (2013), 3410-3418.

[14] W. Li, D.G. Yu, K. Chen, G. Wang and G.R. Williams, Smooth preparation of ibuprofen/zein microcomposites using an epoxy-coated electrospraying head, Mater. Lett. 93 (2013), 125-128.

[15] Y.N. Jiang, H.Y. Mo and D.G. Yu, Electrospun drug-loaded core-sheath PVP/zein nanofibers for biphasic drug release, Int. J. Pharm. 438 (2012), 232- 239.

[16] A.W. Boots, G.R. Haenen and A. Bast, Health effects of quercetin: from antioxidant to nutraceutical, Eur. J. Pharm. 582 (2008), 325-337.

[17] C.C. Chuang, K. Martinez, G. Xie, A. Kennedy, A. Bumrungpert, A. Overman, W. Jia and M.K. McIntosh, Quercetin is equally or more effective than resveratrol in attenuating tumor necrosis factor- $\alpha$-mediated inflammation and insulin resistance in primary human adipocytes, Am. J. Clin. Nut. 92 (2010), 1511-1521.

[18] D.G. Yu, X.Y. Li, W. Chian, Y. Li and X. Wang, The influence of sheath solvents on the quality of ethyl cellulose nanofibers from a modified coaxial electrospinning, Biomed. Mater. Eng. 24 (2014), 695-701.

[19] N.A. Peppas, Analysis of Fickian and non-Fickian drug release from polymers, Pharm. Acta. Hel. 60 (1985), $110-111$.

[20] D.G. Yu, X. Wang, X.Y. Li, W. Chian, Y. Li and Y.Z. Liao, Electrospun biphasic drug release polyvinylpyrrolidone/ethyl cellulose core/sheath nanofibers, Acta. Biomater. 9 (2013), 5665-5672. 